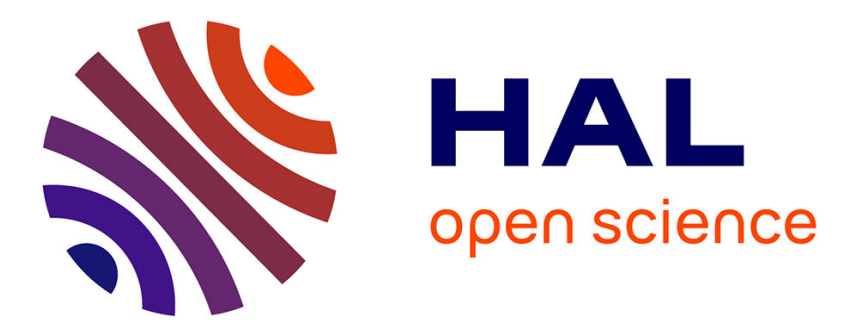

\title{
A Game-Based Self-Organizing Uplink Tree for VoIP Services in IEEE 802.16j Networks
}

Walid Saad, Han Zhu, Merouane Debbah, Are Hjorungnes, Tamer Basar

\section{To cite this version:}

Walid Saad, Han Zhu, Merouane Debbah, Are Hjorungnes, Tamer Basar. A Game-Based SelfOrganizing Uplink Tree for VoIP Services in IEEE 802.16j Networks. International Conference on Communications 2009, Jun 2009, Dresden, Germany. 6 p. hal-00392390

\section{HAL Id: hal-00392390 \\ https://hal-centralesupelec.archives-ouvertes.fr/hal-00392390}

Submitted on 7 Jun 2009

HAL is a multi-disciplinary open access archive for the deposit and dissemination of scientific research documents, whether they are published or not. The documents may come from teaching and research institutions in France or abroad, or from public or private research centers.
L'archive ouverte pluridisciplinaire HAL, est destinée au dépôt et à la diffusion de documents scientifiques de niveau recherche, publiés ou non, émanant des établissements d'enseignement et de recherche français ou étrangers, des laboratoires publics ou privés. 


\title{
A Game-Based Self-Organizing Uplink Tree for VoIP Services in IEEE 802.16j Networks
}

\author{
Walid Saad ${ }^{1}$, Zhu Han ${ }^{2}$, Mérouane Debbah ${ }^{3}$, Are Hjørungnes ${ }^{1}$ and Tamer Başar ${ }^{4}$ \\ ${ }^{1}$ UNIK - University Graduate Center, University of Oslo, Kjeller, Norway, Email: \{saad, areh j\}@unik. no \\ ${ }^{2}$ Electrical and Computer Engineering Department, University of Houston, Houston, USA, Email: zhan2@mail.uh. edu \\ ${ }^{3}$ Alcatel-Lucent Chair in Flexible Radio, SUPÉLEC, Gif-sur-Yvette, France, Email: merouane.debbah@supelec.fr \\ ${ }^{4}$ Coordinated Science Laboratory, University of Illinois at Urbana-Champaign, USA, Email: tbasarecontrol.csl.uiuc.edu
}

\begin{abstract}
In this paper, we propose a game theoretical approach to tackle the problem of the distributed formation of the uplink tree structure among the relay stations (RSs) and their serving base station (BS) in an IEEE 802.16j WiMAX network. Unlike existing literature which focused on the performance assessment of the network in the presence of the RSs, we investigate the topology and dynamics of the tree structure in the uplink of an 802.16j network. We model the problem as a network formation game, with the RSs being the players. In the proposed game, each RS aims to maximize its utility that accounts for the gains from cooperation in terms of bit error rate (BER) and the costs resulting from multi-hop transmission in terms of delay. The proposed utility model is based on the concept of the R-factor which is a parameter suitable for assessing the performance of VoIP services. For forming the tree structure, we propose a distributed myopic best response dynamics in which each RS can autonomously choose the path that connects it to the BS through other relays while optimizing its utility. Using the proposed dynamics, the RSs can self-organize into the tree structure, and adapt this topology to environmental changes such as mobility while converging to a Nash tree network. Simulation results show that the proposed algorithm presents significant gains in terms of average achieved MS utility reaching up to $40.3 \%$ compared to the star topology where all RSs are directly connected to the BS, and up to $42.75 \%$ compared to the case with no RSs. The results also show that the average number of hops in the tree does not exceed 4 even for a network with a large number of RSs.
\end{abstract}

\section{INTRODUCTION}

Distributed spatial diversity using cooperative relaying has been recently proposed as an effective technique for mitigating the fading effects of the wireless channel. By cooperative relaying, several nodes can cooperate with a given source node in the transmission of its data to a far away destination, consequently, providing spatial diversity gains for the source node without the burden of having several antennas physically present on the node. This class of cooperative relaying is known as cooperative communications [1]. It has been shown that by using one or multiple relays [1-3] a significant improvement can be witnessed in terms of bit error rate, throughput or other QoS parameters. Moreover, different aspects of cooperative transmission have been discussed in the literature such as performance analysis [1-3] or resource allocation [4]. Due to this proven advantage of cooperative communications, incorporating relaying into wireless network standards has been under thorough investigation recently. For instance, the IEEE 802.16j mobile multi-hop relay (MMR) task group introduced the concept of multi-hop relaying in the IEEE 802.16 WirelessMAN (WiMAX) family of broadband networks [5]. The main novelty in the standard is the introduction of a

This work was supported by the Research Council of Norway through the projects $183311 / \mathrm{S} 10$ and $18778 / \mathrm{V} 11$. new node, the relay station (RS), for improving the capacity and coverage of 802.16 networks.

Deploying RSs in an 802.16 j network faces several challenging issues. For instance, the authors in [6] maximize the total downlink rate in an $802.16 \mathrm{j}$ network by finding the optimal position of one RS. This optimal RS placement is further studied in [7] for multiple RSs, aiming to maximize the throughput by the usage of the concept of dual relaying. Moreover, the work in [8] provides an algorithm to find the optimal locations of the $\mathrm{BS}$ and the RSs minimizing the cost for deployment of a full scale $802.16 \mathrm{j}$ network. Nonetheless, one challenging area in the context of $802.16 \mathrm{j}$ networks is the design and formation of the tree structure connecting the BS to the RSs in its coverage area as dictated by the standard [5]. Clearly, existing literature focused on the performance of the $802.16 \mathrm{j}$ network and the optimal placement of the RSs, with few work tackling the problem of the formation of the tree structure. The $802.16 \mathrm{j}$ standard states that the tree can be formed either at the BS (centralized approach) or by the sole decisions of the RSs (distributed approach) [5], [9]. The main existing work is in [10] where we presented a distributed network formation game model for forming the uplink tree in an 802.16j network. However, the model in [10] does not account for the costs in terms of the delay incurred by multihop transmission. In order to take into account the delay in the network due to the traffic flow (queueing and transmission delay), a new model and algorithm must be proposed. Another contribution tackling the tree formation in $802.16 \mathrm{j}$ is given in [9] through a centralized approach. However, the work in [9] does not provide a clear algorithm for the tree formation and it does not account for the gains by using cooperative transmission nor for the resulting delay in the network. Finally, a centralized approach can yield some significant overhead and complexity, namely in networks with a rapidly changing environment due to RS mobility or incoming traffic load.

The major contribution of this paper is to provide a distributed algorithm that allows the RSs in an $802.16 \mathrm{j}$ network to autonomously form the uplink tree structure, adapting it to environmental changes as the network evolves. Another main contribution is to model the gains in terms of cooperative transmission, while accounting for the costs in terms of the delay incurred by the multi-hop transmission. For this purpose, we adopt a utility function based on the concept of R-factor, which is a suitable criterion for evaluating the performance of VoIP services [11]. The R-factor provides a utility that ties the packet success rate (PSR) and the delay over a transmission path. For forming the tree, we model the problem as a network formation game and we propose a myopic dynamics in which 
each RS plays its best response strategy that maximizes its utility. The proposed dynamics allows the RSs to self-organize into a Nash network tree structure rooted at the BS. Finally, we illustrate the performance gains of the proposed algorithm through simulations, and we show how the proposed algorithm allows the RSs to autonomously adapt the topology to changes in the environment due to RS mobility or the presence of high traffic.

The rest of this paper is organized as follows: Section II presents the system model and the game formulation. Section III exposes the proposed utility model and presents the proposed dynamics. Simulation results are presented and analyzed in Section IV. Finally, conclusions are drawn in Section V.

\section{System Model And Game Formulation}

\section{A. System Model}

Consider the uplink of an $802.16 \mathrm{j}$ network with $M$ RSs (fixed, mobile or nomadic) and one BS. The 802.16j standard imposes a tree structure to form between the RSs and the BS [5]. Once the uplink tree forms, mobile stations (MSs) can hook to the network by selecting a serving RS (or directly connecting to the BS). Each MS is assigned to the closest RS. We assume that the MSs deposit their packets to their serving RSs using direct transmission. In their turn, the serving RSs act as source nodes transmitting the received MS packets to the BS through one or more hops in the formed tree, using cooperative transmission. This assumption of direct transmission between MS and RS allows to provide a tree formation algorithm that can be easily incorporated in a new or existing $802.16 \mathrm{j}$ network without relying on external entities such as the MSs.

For cooperative transmission between the RSs and the BS, we use the decoded relaying multi-hop diversity channel of [3] whereby each intermediate node on the path between a transmitting RS and the BS combines, encodes and re-encodes the received signal from all preceding terminals before relaying (decode-and-forward). Formally, each MS $k$ in the network is considered as a source of traffic following a Poisson distribution with an average arrival rate $\lambda_{k}$. With such Poisson streams at the entry points of the network (the MSs), we assume that for every RS incoming packets are stored and transmitted in a first in first out (FIFO) fashion and that we have the Kleinrock independence approximation [12, Chap. 3] with each RS being an M/D/1 queueing system. With this approximation, the total traffic that a RS $i$ receives from the MS that it is serving is a Poisson process with an average arrival rate of $T_{i}=\sum_{l=1}^{L_{i}} \lambda_{l}$ where $L_{i}$ is the number of MSs served by RS $i$. Moreover, RS $i$ also receives packets from RSs that are connected to it with a total average rate $R_{i}$. For these $R_{i}$ packets, the sole role of RS $i$ is to relay them to the next hop. In addition, any RS $i$ that has no assigned MSs $\left(L_{i}=0\right.$ and $\left.T_{i}=0\right)$, transmits "HELLO" packets, generated with a Poisson arrival rate of $\eta_{0}$ in order to maintain its link to the BS active during periods of no actual MS traffic. An illustrative example of this model is shown in Fig. 1.

\section{B. Game Formulation and Strategies}

For modeling the interactions among the RSs seeking to form the uplink tree structure, as imposed by the $802.16 \mathrm{j}$ standard,

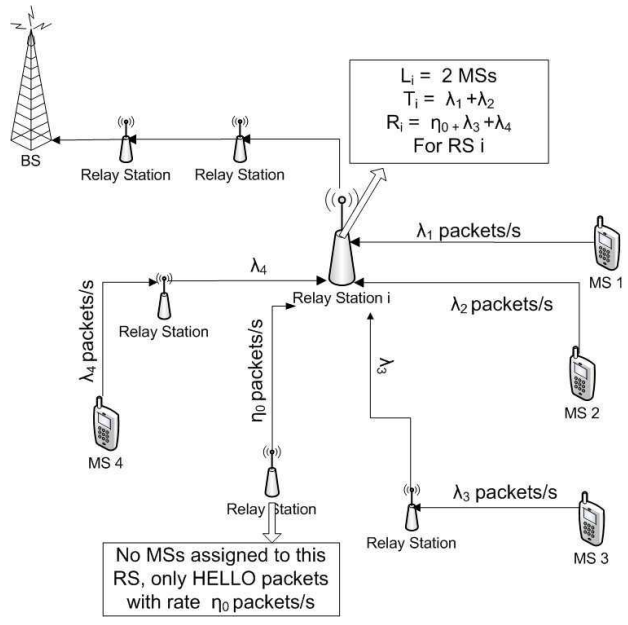

Fig. 1. A prototype of the uplink tree model.

network formation games provide a suitable framework [13-15]. In such games, several independent decision makers (players) interact in order to form a network graph. Depending on the goals of each player, a final network graph $G$, resulting from individual players' decisions, forms. We model the uplink tree formation problem in $802.16 \mathrm{j}$ as a network formation game with the RSs being the players. The result of the interactions among the RSs is a directed graph $G(V, E)$ with $V=\{1, \ldots, M+1\}$ denoting the set of all vertices ( $M$ RSs and the BS) and $E$ denoting the set of all edges (links) between pairs of RSs. Each link between two RSs $i$ and $j$, denoted $i j \in E$, corresponds to an uplink traffic flow between RS $i$ and RS $j$. First and foremost, we define the notion of a path:

Definition 1: A path between two nodes $i$ and $j$ in the graph $G$ is defined as a sequence of nodes $i_{1}, \ldots, i_{K}$ such that $i_{1}=$ $i, i_{K}=j$ and each directed link $i_{k} i_{k+1} \in G$ for each $k \in$ $\{1, \ldots, K-1\}$. We denote the set $Q_{i}$ as the set of all paths from node $i$ to the BS, and thus $\left|Q_{i}\right|$ represents the number of paths from node $i$ to the BS.

As the $802.16 \mathrm{j}$ standard imposes a tree structure between the $\mathrm{RSs}$ and the BS, we adopt the following convention throughout the remainder of this paper

Convention 1: Each RS $i$ is connected to the BS through at most one path, and thus $0 \leq\left|Q_{i}\right| \leq 1, \forall i \in V$. Hence, we denote by $q_{i} \in Q_{i}$ the path between any RS $i$ and the BS.

Finally, we delineate the possible actions or strategies that each RS can take in the proposed network formation game. The strategy space of each RS $i$ consists of the RSs (or the BS) that $i$ wants to connect to. Consequently, the strategy of a RS $i$ is to select the link that it wants to form from the available strategy space. We note that, a RS $i$ cannot connect to a $\mathrm{RS} j$ which is already connected to $i$, in the sense that if $j i \in G$, then ij $\notin G$. Formally, for a current network graph $G$, let $A_{i}=\{j \in$ $V \backslash\{i\} \mid j i \in G\}$ be the set of RSs from which RS $i$ accepted a link $j i$, and $S_{i}=\left\{i j \mid j \in V \backslash\left(\{i\} \bigcup A_{i}\right)\right\}$ as the set of links corresponding to the nodes (RSs or the BS) with whom $i$ wants to connect (note that $i$ cannot connect to RSs that are already connected to it, i.e., RSs in $A_{i}$.). In consequence, the strategy of a RS $i$ is to select the link $s_{i} \in S_{i}$ that it wants to form, i.e., choose the RS that it will connect to. Based on Convention 1, a RS can be connected to at most one other node in our game 
so selecting to form a link $s_{i}$ implicitly implies that RS $i$ will replace its previously connected link (if any) with the new link $s_{i}$.

\section{Network Formation GAme: Utility AND DynAmiCs}

In this section, we derive suitable utilities for modeling the PSR-delay trade off in an $802.16 \mathrm{j}$ network and we construct a dynamics for distributed network tree formation.

\section{A. Utility}

Having modeled the tree formation problem as a network formation game, we propose a utility function that takes into account the QoS in terms of PSR as well as the delay incurred by multi-hop transmission. Consider an actual network tree graph $G$ where each RS extracts a positive utility from the packets successfully transmitted to the BS out of the packets received from the MSs. Each transmitted packet is subject to a bit error rate (BER) due to the transmission over the wireless channel using one or more hops. For instance, for a transmission between a RS $V_{1} \in V$ to a destination $V_{n+1}$ (the destination is always the BS) going through $n-1$ intermediate relays $\left\{V_{2}, \ldots, V_{n}\right\} \subset V$, let $N_{r}$ be the set of all receiving terminals, i.e., $N_{r}=\left\{V_{2} \ldots V_{n+1}\right\}$ and $N_{r(i)}$ be the set of terminals that transmit a signal received by a node $V_{i}$. Hence, for a relay $V_{i}$ on the path from the source $V_{1}$ to the destination $V_{n+1}$, we have $N_{r(i)}=\left\{V_{1}, \ldots, V_{i-1}\right\}$. Given this notation, the BER between a source RS $V_{1} \in V$ and the destination $V_{n+1}=B S$ is computed along the path $q_{V_{1}}=\left\{V_{1}, \ldots, B S\right\} \in Q_{V_{1}}$ using the tight upper bound given in [3, Eq. (10)] for the decoded relaying multi-hop diversity channel with Rayleigh fading and BPSK modulation as follows

$$
\begin{aligned}
P_{q_{V_{1}}}^{e} & \leq \sum_{N_{i} \in N_{r}} \frac{1}{2}\left(\sum _ { N _ { k } \in N _ { r ( i ) } } \left[\prod_{\substack{N_{j} \in N_{r(i)} \\
N_{j} \neq N_{k}}} \frac{\gamma_{k, i}}{\gamma_{k, i}-\gamma_{j, i}}\right.\right. \\
& \left.\left.\times\left(1-\sqrt{\frac{\gamma_{k, i}}{\gamma_{k, i}+2}}\right)\right]\right) .
\end{aligned}
$$

Here, $\gamma_{i, j}=\frac{P_{i} \cdot h_{i, j}}{\sigma^{2}}$ the average received SNR at node $j$ from node $i$ where $P_{i}$ is the transmit power of node $i, \sigma^{2}$ the noise variance and $h_{i, j}=\frac{1}{d_{i, j}^{\alpha}}$ is the path loss with $d_{i, j}$ the distance between $i$ and $j$ and $\alpha$ the path loss exponent. Without loss of generality, we assume that all the RSs transmit with equal power $P_{i}=\tilde{P}, \forall i$. Finally, for a RS $i$ which is connected to the BS through a direct transmission path $q_{i}^{d} \in Q_{i}$ with no intermediate hops, the BER can be given by $P_{q_{i}^{d}}^{e}=\frac{1}{2}\left(1-\sqrt{\frac{\gamma_{i, B S}}{2+\gamma_{i, B S}}}\right)$ [2], [3]; where $\gamma_{i, B S}$ is the SNR received at the BS from RS $i$. Using the BER expression in (1) and with no channel coding, the packet success rate (PSR) $\rho_{i, q_{i}}$ perceived by a RS $i$ over a path $q_{i}$ is defined as follows

$$
\rho_{i, q_{i}}(G)=\left(1-P_{q_{i}}^{e}\right)^{B},
$$

where $B$ is the number of bits per packet. The PSR is a function of the network graph $G$ as the path $q_{i}$ varies depending on how $\mathrm{RS} i$ is connected to the BS in the formed network tree structure.

Transmitting over multi-hop links incurs a significant delay due to buffering and multiple transmission. For this purpose, we consider the average delay $\tau_{i, q_{i}}$ along the path $q_{i}=$ $\left\{i_{1}, \ldots, i_{k}\right\} \in Q_{i}$ from RS $i_{1}=i$ to the BS. A measure of the average delay over $q_{i}$ in a network with Poisson arrivals at the entry points and considering the Kleinrock approximation as in the previous section (each $\mathrm{RS}$ is an M/D/1 queueing system) is given by [12, Chap. 3, Eqs. (3.42), (3.45) and (3.93)]

$\tau_{i, q_{i}}(G)=\sum_{i_{k} i_{k+1} \in q_{i}}\left(\frac{\Lambda_{i_{k}, i_{k+1}}}{2 \mu_{i_{k}, i_{k+1}}\left(\mu_{i_{k}, i_{k+1}}-\Lambda_{i_{k}, i_{k+1}}\right)}+\frac{1}{\mu_{i_{k}, i_{k+1}}}\right)$.

where $\Lambda_{i_{k}, i_{k+1}}=T_{i_{k}}+R_{i_{k}}$ is the total traffic (packets/s) originating from MSs $\left(T_{i_{k}}\right)$ and from RSs $\left(R_{i_{k}}\right)$ traversing link $i_{k} i_{k+1} \in q_{i}$ between $\mathrm{RS} i_{k}$ and $\mathrm{RS} i_{k+1}$. The ratio $\frac{1}{\mu_{i_{k}, i_{k}+1}}$ represents the average transmission time (service time) on link $i_{k} i_{k+1} \in q_{i}$ with $\mu_{i_{k}, i_{k+1}}$ being the service rate on link $i_{k} i_{k+1}$. This service rate is given by $\mu_{i_{k}, i_{k+1}}=\frac{C_{i_{k}, i_{k+1}}}{B}$ where

$$
C_{i_{k}, i_{k+1}}=W \log \left(1+\nu_{i_{k}, i_{k+1}}\right)
$$

is the capacity of the direct transmission between RS $i_{k}$ and RS $i_{k+1}, \nu_{i_{k}, i_{k+1}}=\frac{\tilde{P} h_{i_{k}, i_{k+1}}}{\sigma^{2}}$ is the received SNR from RS $i_{k}$ at $\mathrm{RS} i_{k+1}$, and $W$ is the bandwidth available for $\mathrm{RS} i_{k}$ which is assumed the same for all RSs in the set of vertices $V$, without loss of generality. Similar to the PSR, the delay depends on the paths from the RSs to the BS, hence is a function of the network graph $G$.

For VoIP services, given the delay and the PSR, an appropriate utility function can be defined through the concept of the Rfactor [11]. The R-factor is an expression that links the delay and packet loss to the voice quality [11] as follows

$$
\begin{array}{r}
U_{i}(G)=\Omega_{a}-\epsilon_{1} \tau_{i, q_{i}}(G)-\epsilon_{2}\left(\tau_{i, q_{i}}(G)-\alpha_{3}\right) H-v_{1} \\
-v_{2} \ln \left(1+100 v_{3}\left(1-\rho_{i, q_{i}}(G)\right)\right)
\end{array}
$$

where $\tau_{i, q_{i}}$ is the delay given by (3) expressed in milliseconds, $100 v_{3}\left(1-\rho_{i, q_{i}}\right)$ represents the packet loss percentage (we consider only the packet loss due to errors through $\rho_{i, q_{i}}$ given in (2), ignoring packet loss due to overloaded links). The remaining parameters are constants defined as: $\Omega_{a}=94.2, \epsilon_{1}=0.024$, $\epsilon_{2}=0.11, \epsilon_{3}=177.3, H=0$ if $\tau_{i, q_{i}}<\epsilon_{3}, H=1$ otherwise. $v_{1}, v_{2}$ and $v_{3}$ are parameters dependent on the voice speech codec. The relationship between the R-factor and the speech quality is such that as the R-factor increases, the voice quality improves. For different voice codecs, different R-factor ranges provide an indication on the voice quality varying from poor, low, medium, high to best as the R-factor increases [11].

Although the RSs are the players of the game, the final results must assess the performance of the MSs in terms of the R-factor achieved (considered as MS utility). For computing the R-factor of the MSs, the PSR and the delay for the whole transmission from MS to BS must be considered. For instance, based on the proposed network model in Section II, the PSR perceived by each MS $i$ served by a RS $j$ is given by

$$
\zeta_{i, j}(G)=\rho_{i, i j} \cdot \rho_{j, q_{j}}(G),
$$

where $\rho_{i, i j}$ is the PSR on the direct transmission between MS $i$ and RS $j$ (independent of network graph $G$ ) and $\rho_{j, q_{j}}(G)$ is the PSR from RS $j$ to the BS along path $q_{j}$ given by (2) (which can be either a multi-hop transmission or a direct transmission depending on how $\operatorname{RS} j$ is connected in the tree graph $G$ ). 
Moreover, the delay perceived by a MS $i$ served by $\mathrm{RS} j$ is given by (3) by taking into account, in addition to the delay on the path $q_{j}$, the traffic on the link $i j$ between the MS and the RS, i.e., the buffering and transmission delay at the MS level. Having the PSR given by (6) and the delay, the utilities of the MSs can be computed for performance assessment.

\section{B. Dynamics of Network Formation}

Having formulated a network formation game between the RSs, the next step is to define an algorithm of interaction between the RSs in order to form the required network graph. Network formation algorithms are referred to in the literature as dynamics of network formation. For our game, prior to providing a dynamics for network formation, we first show that any dynamics will result in a connected tree structure, as follows

Property 1: Any network graph $G$ resulting from a dynamics applied to the proposed network formation game is a connected directed tree structure rooted at the BS.

Proof: By inspecting the utility in (5), we notice that if a RS $i$ is not connected to the BS through direct transmission or other multi-hop paths, the BER will be $P_{q_{i}}^{e}=1$ since $Q_{i}=\emptyset$ (no path from the RS $i$ to the BS). Consequently, for the utility in (5) the last term will be maximized since $\rho_{i, q_{i}}=0$ in this case and thus the whole utility will be minimized. In other words, a disconnection by any RS drastically decreases its utility. Hence, there is no incentive for any RS to disconnect from the BS and any graph $G$ formed using the proposed game will be a connected graph. Moreover, due to Convention 1, the connected graph is thus a tree rooted at the BS.

Due to the high disconnection cost, if a RS is unable to find any partner suitable for forming a link, it will connect to the BS through direct transmission. Thus, our network initially starts with all the RSs connected to the BS (star topology), before engaging in the network formation game.

Denote $G_{s_{i}, \boldsymbol{s}_{-i}}$ as the graph $G$ formed when RS $i$ plays a strategy $s_{i} \in S_{i}$ while all other RSs maintain their vector of strategies $s_{-i}=\left[s_{1}, \ldots, s_{i-1}, s_{i+1}, \ldots, s_{M}\right]$. We define the best response for a RS as follows [15].

Definition 2: A strategy $s_{i}^{*} \in S_{i}$ is a best response for a RS $i \in V$ if $U_{i}\left(G_{s_{i}^{*}, s_{-i}}\right) \geq U_{i}\left(G_{s_{i}, s_{-i}}\right), \forall s_{i} \in S_{i}$. Thus, the best response for $\mathrm{RS} i$ is to make the selection of the link that maximizes its utility given that the other RSs maintain their vector of strategies.

By using the different properties of the RS network formation game, we propose best response dynamics that allow a distributed formation of the network graph. The proposed dynamics assume that each RS is myopic, in the sense that the RSs aim at improving their payoff considering only the current state of the network without taking into account the future evolution of the network. Finding an optimal dynamics is a very complex problem, and there exist no strict rules for the network formation dynamics in the literature [13]. Therefore for each network formation game model, different dynamics rules must be applied suited to the considered model. Network formation literature encompasses several myopic dynamics for various game models with directed and undirected graphs [13-15]. In this paper, we propose a myopic dynamics inspired from [13] and [15]. The
TABLE I

PROPOSED NETWORK FORMATION DYNAMICS

Initial State
All the RSs start by directly connecting to the BS in a star topology.
Two phases in each round of the dynamics
Phase 1 - Fair Prioritization:
Prioritize the RSs from the highest to the lowest current BER.
Phase 2 - Myopic Dynamics:
The RSs take action sequentially by priority.
Each RS $i$ plays its best response $s_{i}^{*}$, maximizing its
utility (R-factor).
The best response $s_{i}^{*}$ of each RS is a replace link
operation through which a RS $i$ splits from its current parent
RS and replaces it with a new RS that maximizes its utility.
Multiple rounds are run until convergence to the final Nash tree
$G^{\dagger}$ where no RS can improve its utility by a unilateral change of
strategy.

proposed algorithm is composed of several rounds where each round consists mainly of two phases: a fair prioritization phase and a dynamics phase. In the fair prioritization phase, we propose a priority function that assigns a priority to each RS. In the dynamics phase, by increasing priority order, the RSs are allowed to interact.

Therefore, each round of the proposed algorithm begins with the fair prioritization phase where each RS is assigned a priority depending on its actual perceived BER: RSs with a higher BER are assigned a higher priority. The motivation behind this procedure is to fairly allow RSs that are perceiving a bad channel to possess an advantage in selecting their partners; for the purpose of improving their BER. Thus, the RSs experiencing a high BER can select their partners out of a larger space of strategies during the dynamics phase. Other priority functions can also be used, and in a general case, a random priority function can be defined. Following prioritization, the RSs start selecting their strategies sequentially by order of priority. During its turn, each RS $i$ chooses to play its best response $s_{i}^{*} \in S_{i}$ in order to maximize its utility at each round given the current network graph resulting from the strategies of the other RSs. The best response of each RS can be seen as a replace operation, whereby the RS will replace its current link to the BS with another link that maximizes its utility (if such a link is available).

Multiple rounds consisting of the above two phases will be run until convergence to the final tree structure $G^{\dagger}$ where the RSs can no longer improve their utility through best responses. The proposed algorithm is summarized in Table I.

The stability of the final graph $G^{\dagger}$ is given using the concept of Nash equilibrium applied to network formation games [15]

Definition 3: A network graph $G$ in which no node $i$ can improve its utility by a unilateral change in its strategy $s_{i} \in S_{i}$ is a Nash network.

Therefore, a Nash network is a network where the links chosen by each node are the best responses. The Nash network is, thus, a network where the nodes are in a Nash equilibrium with no node able to improve its utility by unilaterally changing its current strategy. When our dynamics converge, and as an immediate consequence of playing a best response dynamics, we have

Lemma 1: The final tree structure $G^{\dagger}$ resulting from the proposed dynamics is a Nash network. 


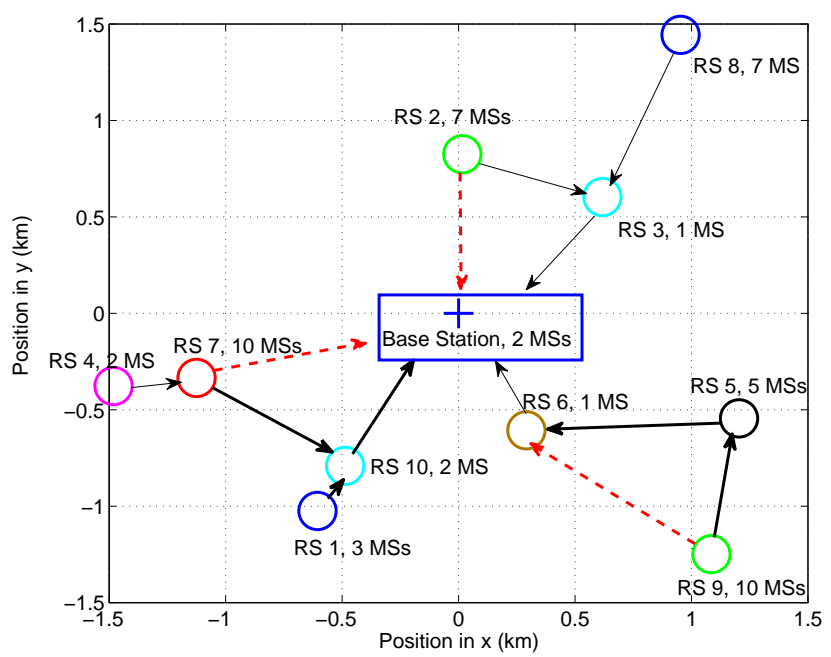

Fig. 2. Snapshot of a tree topology formed using the proposed algorithm with 10 RSs before (solid line) and after (dashed line) the random deployment of 50 MSs (MS positions not shown for clarity).

\section{Simulation Results And Analysis}

For simulations, we consider a square area of $4 \mathrm{~km} \times 4 \mathrm{~km}$ with the BS at the center. We deploy the RSs and the MSs within this area. The transmit power is $\tilde{P}=50 \mathrm{~mW}$ (RSs and MSs), the noise level is $-110 \mathrm{dBm}$, and the bandwidth per RS is set to $W=100 \mathrm{kHz}$. For path loss, we set the propagation loss to $\alpha=3$. For the VoIP traffic, we consider a traffic of $64 \mathrm{kbps}$, divided into packets of length $B=200$ bits with an arrival rate of 320 packets/s. For the HELLO packets we set $\eta_{0}=1$ packet/s with the same packet length of $B=200$ bits. Finally, without loss of generality, we select the G.729 codec for voice, hence $v_{1}=12, v_{2}=15$ and $v_{3}=0.6[11]$.

In Fig. 2 we randomly deploy $M=10 \mathrm{RSs}$ within the BS area. For clarity of the figure, we show only the area around the BS which contains RSs. The network formation game starts with the star topology where all RSs are connected directly to the BS. Prior to the presence of the MSs in the network (only HELLO packets present), the RSs interact and converge to a final Nash tree structure shown by the solid lines in the figure. This figure clearly shows how the RSs connect to their nearby partners, forming the tree structure. Furthermore, we randomly deploy $50 \mathrm{MSs}$ in the area, and show how the RSs autonomously adapt the topology to this incoming traffic. The new resulting network structure upon deployment of MSs is shown in dashed lines. The RSs autonomously self-organize and adapt to the deployed traffic. For instance, RS 3 can no longer accommodate the traffic generated by RS 2 as it drastically decreases its utility. As a result, RS 2 takes the decision to disconnect from RS 3 and improve its R-factor by directly connecting to the BS. Similarly, RS 7 disconnects from RS 10 and connects directly to the BS. Moreover, RS 9 finds it beneficial to replace its link with RS 5 with a link with the less loaded RS 6. In a nutshell, Fig. 2 summarizes the operation of the proposed adaptive dynamics with and without the presence of external MS data traffic.

Furthermore, we assess the effect of mobility on the network structure. We consider the network of Fig. 2 prior to the deployment of the MSs and we assume that RS 5 is moving

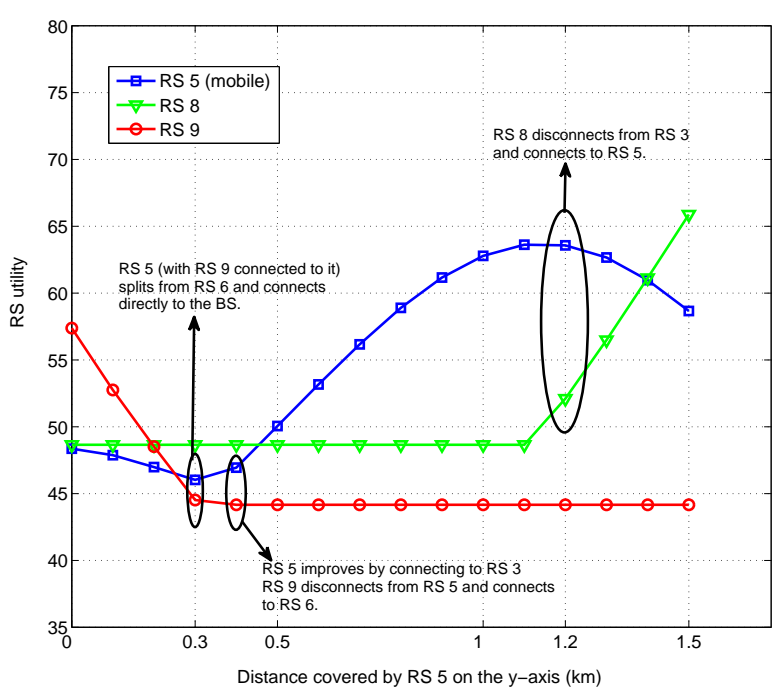

Fig. 3. Self-adaptation of the network's tree topology to mobility shown through the variation of the utility of RS 5 as it moves upwards on the y-axis prior to any MS presence.

upwards on the positive $y$-axis while the other RSs remain fixed. The changes in utility of the concerned RSs during the movement of RS 5 are shown in Fig. 3. As RS 5 moves upwards, its utility starts by dropping since the distance to its serving RS (RS 6) increases. Upon moving $0.3 \mathrm{~km}$, RS 5 finds it beneficial to replace its link with RS 6 with a direct link to the BS, adapting the topology. Meanwhile, RS 9 decides to remain connected to RS 5 since it cannot improve its utility otherwise. However, when RS 5 has moved $0.4 \mathrm{~km}$, RS 9 decides to disconnect from RS 5 and connect directly to RS 6 improving its utility. This decreases the traffic on RS 5 which is now closer to RS 3 and can maximize its utility by connecting to RS 3. As RS 5 gets closer to RS 3, its utility continues to improve. After moving $1.2 \mathrm{~km}$, RS 5 approaches RS 8 which finds it beneficial to disconnect its link with RS 3 and connect to RS 5. At this point the utility of RS 5 drops due to the newly accepted traffic; moreover, this utility drops further as RS 5 distances itself from its serving RS (RS 3). The utility of RS 8 continues to improve as RS 5 gets closer to it. Through these results, we clearly illustrate how the RSs can autonomously self-organize adapting the topology to mobility. Similar results can be shown when new RSs enter the network or RSs leave the network with or without the presence of MSs, but these results are omitted due to space limitation.

In Fig. 4, we show the average achieved utility per MS as the number of RSs, $M$, in the network increases. The results are averaged over random positions of the MSs and the RSs in a network having $40 \mathrm{MSs}$. We compare the performance of the proposed algorithm against the star topology whereby each RS is directly connected to the BS as well as the scenario where no RSs exist in the network. In this figure, we clearly see that as the number of RSs in the network increases the performance of the proposed algorithm as well as for the star topology improves. However, for the star topology the slope of increase is much slower than that of the proposed algorithm. In addition, the proposed algorithm presents a clear performance advantage, increasing with the number of the RSs, and reaching up to $40.3 \%$ and $42.75 \%$ (at $M=25 \mathrm{RSs}$ ) relative to the star 


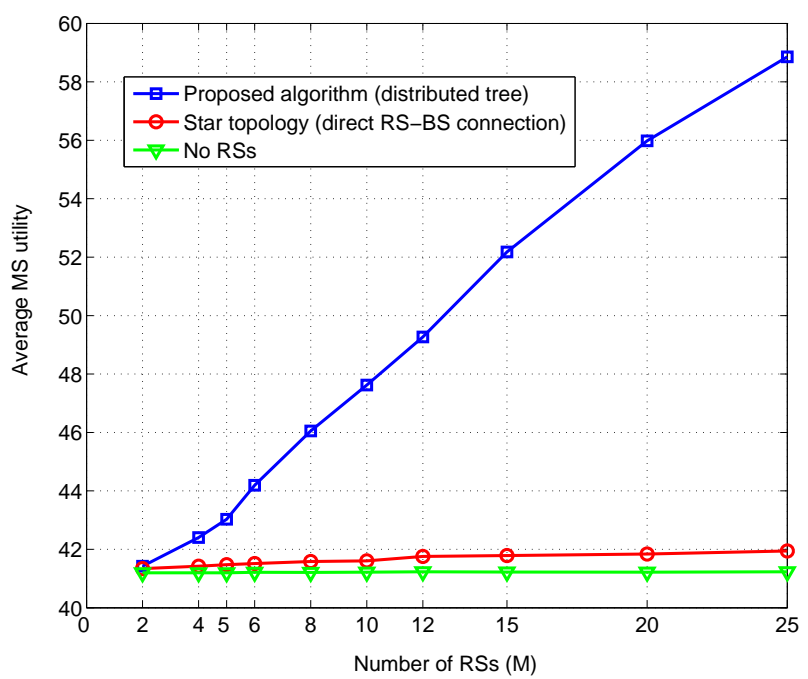

Fig. 4. Performance assessment of the proposed distributed tree algorithm for a network with $40 \mathrm{MSs}$ shown through the average achieved MS utility vs. number of RSs $M$ in the network (average over random positions of MSs and RSs).

topology and the no RSs case, respectively.

In Fig. 5, we plot the average $M_{\mathrm{av}}$ and maximum $M_{\max }$ number of hops achieved in the network tree as the number of RSs increases for a network with 40 MSs (results are averaged over random positions of MSs and RSs). It must be noted that the plotted number of hops corresponds to the RS-RS or RS-BS links in the tree, without accounting for the entry point MS-RS hop. First and foremost, as the number of RSs $M$ in the network increases both the average and the maximum number of hops increase. However, we note that, almost 5 additional RSs must be deployed before the average and the maximum number of hops increase of 1 . This result shows that, due to the delay cost for multi-hop transmission, the number of hops in the tree do not increase drastically with the number of RSs in the network. In general, we can easily remark that the increase slope of $M_{\mathrm{av}}$ and $M_{\max }$ with respect to the number of RSs $M$ is small. Finally, we note that the maximum number of hops reaches 7 hops while the average number of hops does not exceed 4 hops, even for a network with $M=25$ RSs.

\section{CONCLUSions}

This paper proposed a novel distributed approach to the uplink tree formation problem in $802.16 \mathrm{j}$ networks through a network formation game model. In the proposed game, the RSs engage in pairwise interactions for forming directed links that result in a final uplink tree structure rooted at the BS. Each RS aims to maximize its utility which accounts for the gains in terms of PSR while taking into account the delay costs incurred by multi-hop transmission. For VoIP services, a suitable utility is derived through the concept of the R-factor which is a parameter that assesses VoIP quality through an expression that ties the PSR and the delay. We proposed a best response dynamics for network formation that converges to a Nash network. Using the proposed dynamics, the RSs are able to autonomously form the tree topology and adapt it to environmental changes such as mobility or incoming traffic. Simulation results show that the proposed algorithm presents significant gains in terms of average achieved MS utility reaching up to $40.3 \%$ improvement

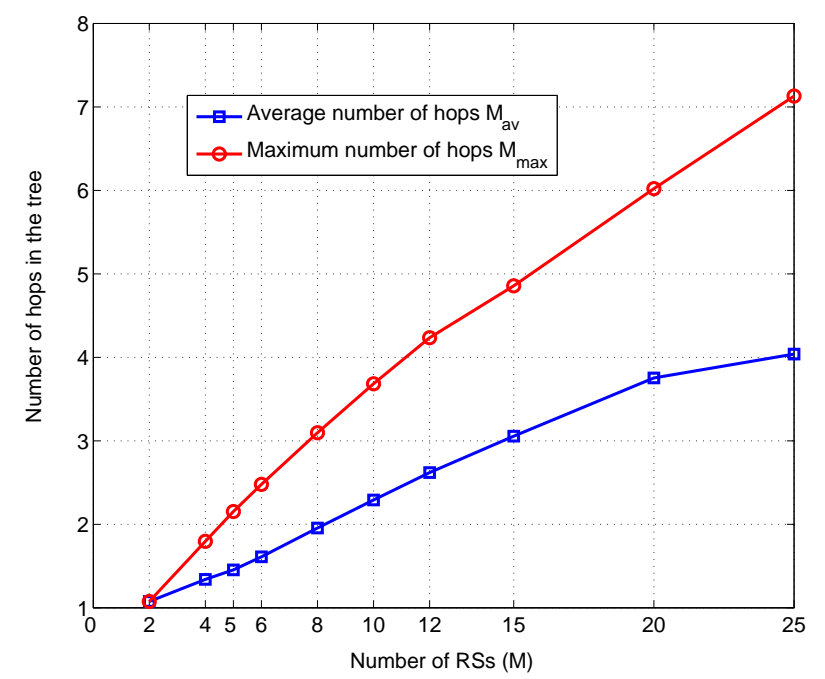

Fig. 5. Average and maximum number of hops in the final tree structure for a network with 40 MSs vs. number of RSs $M$ in the network (average over random positions of MSs and RSs).

compared to the star topology (all RSs directly connected to the BS), and up to $41.75 \%$ compared to the case with no RSs. The results also show that the average number of hops in the tree does not exceed 4 even for networks having a large number of RSs.

\section{REFERENCES}

[1] J. Laneman, D. Tse, and G. Wornell, "Cooperative diversity in wireless networks: efficient protocols and outage behavior," IEEE Trans. Inform. Theory, vol. 50, pp. 3062-3080, Dec. 2004.

[2] A. Sadek, W. Su, and K. R. Liu, "Multinode cooperative communications in wireless networks," IEEE Trans. Signal Processing, vol. 55, pp. 341-355, Jan. 2007.

[3] J. Boyer, D. Falconer, and H. Yanikomeroglu, "Multihop diversity in wireless relaying channels," IEEE Trans. Commun., vol. 52, pp. 18201830 , Oct. 2004

[4] Z. Han and K. J. Liu, Resource allocation for wireless networks: basics, techniques, and applications. Cambridge University Press, 2008.

[5] The Relay Task Group of IEEE 802.16, "The p802.16j baseline document for draft standard for local and metropolitan area networks," $802.16 \mathrm{j}$ 06/026r4, Tech. Rep., Jun. 2007.

[6] B. Lin, P. Ho, L. Xie, and X. Shen, "Optimal relay station placement in IEEE 802.16j networks," in Proc. International Conference on Communications and Mobile Computing, Hawaii, USA, Aug. 2007.

[7] - "Relay station placement in IEEE $802.16 \mathrm{j}$ dual-relay MMR networks," in Proc. Int. Conf. on Communications, Beijing, China, May 2008.

[8] Y. Yu, S. Murphy, and L. Murphy, "A clustering approach to planning base station and relay station locations in IEEE 802.16j multi-hop relay networks," in Proc. Int. Conf. on Communications, Beijing, China, May 2008.

[9] H. Lee, H. Park, Y. Choi, Y. Chung, and S. Rhee, "Link adaptive multihop path management for IEEE 802.16j," in IEEE C802/16j-07/1053, Jan. 2007.

[10] W. Saad, Z. Han, M. Debbah, and A. Hjørungnes, "Network formation games for distributed uplink tree construction in IEEE 802.16j networks," in Proc. IEEE Global Communication Conference, New Orleans, LA, USA, Dec. 2008.

[11] ITU-T Recommendation G.107, "The emodel, a computational model for use in transmission planning," ITU-T, Tech. Rep., Jun. 2002.

[12] D. Bertsekas and R. Gallager, Data networks. New Jersey, USA: Prentice Hall, Mar. 1992.

[13] G. Demange and M. Wooders, Group formation in economics: networks, clubs and coalitions. Cambridge, UK: Cambridge University Press, Mar. 2005.

[14] R. Johari, S. Mannor, and J. Tsitsiklis, "A contract based model for directed network formation," Games and Economic Behavior, vol. 56, pp. 201-224, Jul. 2006.

[15] J. Derks, J. Kuipers, M. Tennekes, and F. Thuijsman, "Local dynamics in network formation," in Proc. Third World Congress of The Game Theory Society, Illinois, USA, Jul. 2008. 